\title{
Good evidence for effectiveness of analgesics most commonly prescribed by UK dentists
}

\author{
What is the relative efficacy of single-dose oral analgesics after third molar \\ extraction?
}

\begin{abstract}
Barden J, Edwards JE, McQuay HJ, Wiffen PJ, Moore RA. Relative efficacy of oral analgesics after third molar extraction. Br Dent $J$ 2004; 197:407-411

Data sources Dental trials were sought among systematic reviews of randomised, double-blind studies of analgesics in acute pain, which were identified from the Cochrane Library, Biological Abstracts, MEDLINE, PubMed and the Oxford Pain Relief database.

Data extraction and synthesis Data were extracted independently by two reviewers. Dichotomous information from active and placebo treatments was used, first to calculate the statistical significance using relative risk, and then to evaluate the clinical relevance using number-needed-to-treat (NNT).

Results Nonsteroidal anti-inflammatory drugs (NSAID) and cyclooxygenase (COX)-2 inhibitors had the lowest (best) NNT for the outcome of pain at least halved over $4-6 \mathrm{~h}$ compared with placebo. With the best performing analgesics, 50-70 patients out of 100 had good pain relief compared with about 10 out of 100 with placebo. Only paracetamol $(600 / 650 \mathrm{mg})$ plus codeine $(60 \mathrm{mg})$ was associated with any significant increase in any patient experiencing an adverse event.

Conclusions NSAID and COX-2 inhibitors have the lowest (best) NNT. These may also have fewer adverse effects after third molar surgery, but conclusive evidence is lacking. At least $80 \%$ of analgesic prescribing by UK dentists is in line with the best available evidence on efficacy and safety.
\end{abstract}

\section{Commentary}

One of the questions most commonly asked by dentists is about the efficacy of analgesic treatments in dental pain. This paper is based on single-dose trials of oral analgesics after third molar extractions, thus providing information on the relative efficacy of certain doses, but not fixed regimens, of analgesics.

It is very interesting to note that the lowest (best) NNT were for nonselective COX inhibitors (termed NSAID in the paper) and selective COX-2 inhibitors (eg, the coxibs ) at standard or high doses. Examples to illustrate this point are valdecoxib $40 \mathrm{mg}$ and diclofenac $100 \mathrm{mg}$ (both NNT of 1.6), and rofecoxib $50 \mathrm{mg}$ and diclofenac $50 \mathrm{mg}$ (both NNT of 2.1); these were followed by ibuprofen $400 \mathrm{mg}$ (NNT of 2.2). These results offer intriguing arguments for the debate over which factors (ie, potency of inhibition, selectivity of inhibition and/or pharmacokinetic properties) really cause the clinical effective analgesic effect of these drugs.

The results shown in the paper, however, may attract clinicians to make uncritical increases of the standard recommended doses of nonselective COX inhibitors without considering the possible relationship with increased incidence of adverse effects of these drugs. This is a real possibility: the negative adverse-effect profile of the coxibs on the cardiovascular system after long-term use has resulted in some of them being withdrawn from the market.

Address for correspondence: RA Moore, Research Director, Pain Research and Nuffield Department of Anaesthetics, University of Oxford, Oxford Radcliffe Hospital, The Churchill, Headington, Oxford OX3 7LJ, UK. E-mail: andrew.moore@pru.ox.ac.uk
It is disappointing that the paper does not present more specific information on the incidence, following the use of COX inhibitors after third molar extractions, of alveolitis sicca dolorosa (dry socket) and other adverse effects of interest to dentists. Irrespective of their selectivity, differences between COX inhibitors in their adverseeffect profiles, especially regarding dry socket, would be of primary importance in a dentist's choice of analgesic treatment, at least after tooth extractions.

The present paper provides some reasonable and required answers to the question about efficacy of single-dose analgesics in dental pain. At least one small question remains unanswered after reading this paper, however. Why have the authors omitted paracetamol $1000 \mathrm{mg}$ plus codeine $60 \mathrm{mg}$ from their meta-analyses?

Dr RA Moore responds: The adverse events reported in these single-dose trials are those identified in our analysis of the studies available and this includes dry socket and others. The fact that adverse events are not better reported in the original studies is a topic on which we have published previously. ${ }^{1,2}$ The main point of these meta-analyses is to demonstrate how studies may be better performed in future, and we have a recent publication on this topic. ${ }^{3}$

As the possible negative cardiovascular outcomes of coxibs postdate the submission of our review to the British Dental Journal in early 2003, it was difficult to include this in the discussion. Moreover, the current evidence is that cardiovascular effects of coxibs seen in cancer or precancer are also shared by aspirin, and that any increased cardiovascular risk in arthritis is shared with aspirin and NSAIDs. An electronic update of the Textbook of Pain (www.textbookofpain.com/) to be launched in August has an essay from us on the topic.

The question of paracetamol $1000 \mathrm{mg}+$ codeine $60 \mathrm{mg}$ is a difficult one. We approached this in a systematic review looking at different sources of evidence a while ago. ${ }^{4}$ The problem was that there were only two trials, with only 77 patients with dental surgery receiving this combination, and with unbalanced numbers that can skew statistics. We chose to omit it, having made a defence of the combination based on a wider body of evidence than just these two trials earlier.

\section{Lasse A Skoglund}

Section of Dental Pharmacology and Pharmacotherapy, Faculty of Dentistry, University of Oslo, Oslo, Norway

1. McQuay HJ, Edwards JE, Moore RA. Evaluating analgesia: the challenges. Am J Ther 2002; 9:179-187.

2. Edwards JE, McQuay HJ, Moore RA, Collins SL. Reporting of adverse effects in clinical trials should be improved: lessons from acute postoperative pain. J Pain Symptom Manage 1999; 18:427-437.

3. Moore RA, Edwards JE, McQuay HJ. Acute pain: Individual patient meta-analysis shows the impact of different ways of analysing and presenting results. Pain 2005; 116:322-331.

4. Smith LA, Moore RA, McQuay HJ, Gavaghan D. Using evidence from different sources: an example using paracetamol $1000 \mathrm{mg}$ plus codeine $60 \mathrm{mg}$. BMC Med Res Methodol 2001; 10:1:1.

Evidence-Based Dentistry (2005) 6, 66.

doi:10.1038/sj.ebd.6400337 